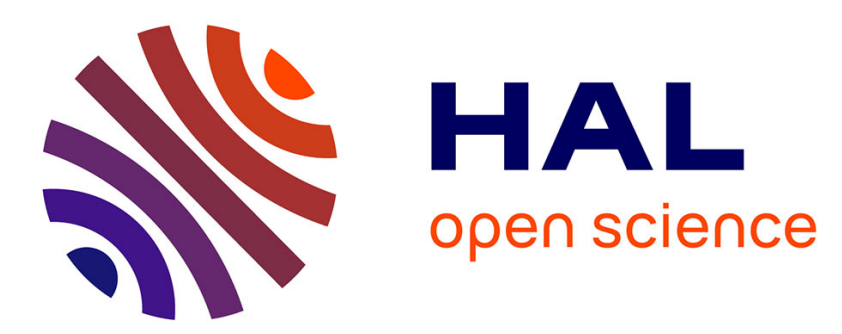

\title{
Funclust: a curves clustering method using functional random variables density approximation
}

\author{
Julien Jacques, Cristian Preda
}

\section{To cite this version:}

Julien Jacques, Cristian Preda. Funclust: a curves clustering method using functional random variables density approximation. Neurocomputing, 2013, 112, pp.164-171. 10.1016/j.neucom.2012.11.042 . hal-00628247v2

\section{HAL Id: hal-00628247 \\ https://hal.science/hal-00628247v2}

Submitted on 13 Oct 2012

HAL is a multi-disciplinary open access archive for the deposit and dissemination of scientific research documents, whether they are published or not. The documents may come from teaching and research institutions in France or abroad, or from public or private research centers.
L'archive ouverte pluridisciplinaire HAL, est destinée au dépôt et à la diffusion de documents scientifiques de niveau recherche, publiés ou non, émanant des établissements d'enseignement et de recherche français ou étrangers, des laboratoires publics ou privés. 


\title{
Funclust: a curves clustering method using functional random variables density approximation
}

\author{
Julien Jacques and Cristian Preda \\ Laboratoire Paul Painlevé, UMR CNRS 8524, University Lille I, Lille, France \\ MODAL team, INRIA Lille-Nord Europe and Polytech'Lille
}

\begin{abstract}
A new method for clustering functional data is proposed under the name Funclust. This method relies on the approximation of the notion of probability density for functional random variables, which generally does not exists. Using the Karhunen-Loeve expansion of a stochastic process, this approximation leads to define an approximation for the density of functional variables. Based on this density approximation, a parametric mixture model is proposed. The parameter estimation is carried out by an EM-like algorithm, and the maximum a posteriori rule provides the clusters. The efficiency of Funclust is illustrated on several real datasets, as well as for the characterization of the Mars surface.
\end{abstract}

Keywords: Functional data, model-based clustering, random variable density, functional principal component analysis.

\section{Introduction}

Cluster analysis aims to identify homogeneous groups of data without using any prior knowledge on the group labels of data. Several methods, from hierarchical clustering [1] or k-means [2] to more recent probabilistic model-based clustering algorithms [3, 4] have been proposed. A particular type of data for which clustering is a difficult task is the functional data (curves or trajectories [5]). The main difficulty in clustering such data arises because of the infinite dimensional space that data belong to.

Consequently, most of clustering algorithms for functional data consists in a first step of transforming the infinite dimensional problem into a finite dimensional one and in a second step, using a model-based clustering method 
designed for finite dimensional data. The representation of functions in a finite dimensional space can be carried out in several ways: discretizing the time interval, approximating data into a finite basis of functions or using some dimension reduction techniques such as functional principal component analysis (FPCA, [5]). Note that using time interval discretization, we need to observe all curves at the same time stamps. The size of discretization being generally large, regularized clustering algorithm should be used [3, $4,6,7,8]$. The approximation of data (curves) into a finite dimensional space of functions - using a basis of functions such as spline or Fourier - has the advantage to take into account possible measurement errors. Indeed, in presence of such errors, a least square approximation approach can be used to estimate the coefficients of the basis approximation, whereas an interpolation method can be used if the data are observed without noise. More about smoothing functional data is presented in [5].

In the framework of clustering, the main contributions use the k-means algorithm, applied on a $B$-spline fitting [9], on defined principal points of curves [10], on the truncated Karhunen-Loeve expansion [11] or more recently on wavelets [12]. As in the finite dimensional setting, where Gaussian modelbased clustering generalizes the k-means algorithm, some other works introduce more sophisticated model-based techniques: [13] define an approach particularly effective for sparsely sampled functional data, [14] propose a nonparametric Bayes wavelet model for clustering of functional data based on a mixture of Dirichlet processes, [15] build a specific clustering algorithm based on parametric time series models, [16] extend the high-dimensional data clustering algorithm (HDDC, [7]) to the functional case.

In the finite dimensional setting, model-based clustering algorithms assume that the data is sampled from a mixture of probability densities. This is not directly applicable to functional data since the notion of probability density generally does not exist for functional random variable. Consequently, model-based clustering algorithms previously cited assume a parametric distribution on a finite series of coefficients characterizing the curves.

In the present paper, we use the density approximation defined in [17] to build our model-based clustering. This density approximation, based on the truncation of the Karhunen-Loeve expansion, relies on the probability density of the first principal components [5] of the curves. Our model assumes a cluster-specific Gaussian distribution for the principal component scores. The number of principal components as well as the computation of the principal component scores are cluster specific. 
The most related methods are the k-centres algorithm $(k C F C,[11])$ and the FunHDDC algorithm [16]. In [11], the k-means algorithm is based on the distance between the truncated Karhunen-Loeve expansion of the curves. As for our model, different truncation orders are allowed for each cluster. But, contrary to our model, the k-means algorithm assumed equal within-cluster variations. Moreover, the estimation algorithm used in k-means performed classification at each iteration, whereas only a fuzzy partition is used in our algorithm. These differences are similar to the differences between k-means and more general Gaussian mixture models: k-means assumes equal diagonal covariance matrices for each cluster, whereas Gaussian mixture models allow more general covariance structures; k-means uses a CEM algorithm (Classification Expectation Maximisation, [18]) whereas Gaussian mixture models are generally estimated more efficiently by the EM algorithm (Expectation Maximisation, [19]). In [16], the authors assume a parsimonious Gaussian model on the principal component scores issued from cluster-specific functional principal components analysis (FPCA). Real-data applications (Section 4) will illustrate numerically these differences between our method, kCFC and FunHDDC.

The paper is organized as follows. Section 2 presents the approximation for the probability density of a functional random variable introduced in [17]. Model-based clustering using this approximation as well as the model estimation procedure, based on the EM algorithm, are presented in Section 3. Finally, Section 4 compares our method with other clustering algorithms on real datasets. An application to the characterization of the surface of Mars using clustering of spectrum concludes the paper.

\section{Density approximation for functional data}

Let $X$ be a functional random variable with values in $L_{2}([0, T]), T>0$, and assume that $X$ is a $L_{2}$-continuous stochastic process, $X=\{X(t), t \in$ $[0, T]\}$. Let $\underline{X}=\left(X_{1}, \ldots, X_{n}\right)$ be an i.i.d sample of size $n$ from the same probability distribution as $X . \underline{X}$ is generally called a sample of functional data for which the underlying model is $X$.

It is well known that the notion of probability density for this type of random variables is not well defined. In [20] a non parametric approach for the estimation of probability density is presented as an extension of the multivariate finite case. This non parametric approximation is not helpful in the context of model-based approaches. 
Our work is based on the idea developed in [17] where an "approximation density" for $X$ is proposed using the Karhunen-Loeve expansion (or principal component analysis (PCA)):

$$
X(t)=\mu(t)+\sum_{j=1}^{\infty} C_{j} \psi_{j}(t)
$$

where $\mu$ is the mean function of $X, C_{j}=\int_{0}^{T}(X(t)-\mu(t)) \psi_{j}(t) d t, j \geq 1$, are zero-mean random variables (called principal components) and $\psi_{j}$ 's form an orthonormal system of eigen-functions of the covariance operator of $X$ :

$$
\int_{0}^{T} \operatorname{Cov}(X(t), X(s)) \psi_{j}(s) d s=\lambda_{j} \psi_{j}(t), \forall t \in[0, T] .
$$

Notice that the principal components $C_{j}$ 's are uncorrelated random variables of variance $\lambda_{j}$. Considering the principal components indexed upon the descending order of the eigenvalues $\left(\lambda_{1} \geq \lambda_{2} \geq \ldots\right)$, let $X^{(q)}$ denotes the approximation of $X$ by truncating (1) at the $q$ first terms, $q \geq 1$,

$$
X^{(q)}(t)=\mu(t)+\sum_{j=1}^{q} C_{j} \psi_{j}(t) .
$$

Then, $X^{(q)}$ is the best approximation of $X$, under the mean square criterion, among all the approximations of the same type (linear combination of $q$ deterministic functions of $t$ with random coefficients, [21]). Denoting by $\|$. the usual norm on $L_{2}([0, T])$, we have

$$
\mathbb{E}\left(\left\|X-X^{(q)}\right\|^{2}\right)=\sum_{j \geq q+1} \lambda_{j} \quad \text { and } \quad\left\|X-X^{(q)}\right\| \underset{q \rightarrow \infty}{\stackrel{\text { m.s. }}{\longrightarrow}} 0 .
$$

Without loss of generality, we will suppose in the following that $X$ is a zeromean stochastic process, i.e. $\mu(t)=0, \forall t \in[0, T]$.

Based on the approximation of $X$ by $X^{(q)}$, in [17] it is shown that the probability of $X$ to belong to a ball of radius $h$ centred in $x \in L_{2}[0, T]$ can 
be written as

$$
\log P(\|X-x\| \leq h)=\sum_{j=1}^{q} \log f_{C_{j}}\left(c_{j}(x)\right)+\xi(h, q(h))+o(q(h)),
$$

where $f_{C_{j}}$ is the probability density of $C_{j}$ and $c_{j}(x)$ is the $j$ th principal component score of $x, c_{j}(x)=<x, \psi_{j}>_{L_{2}}$. The functions $q$ and $\xi$ are such that $q$ grows to infinity when $h$ decreases to zero and $\xi$ is depending only on $h$. Thus, the dependency of $\log P(\|X-x\| \leq h)$ with $x$ is contained in the term $\sum_{j=1}^{q} \log f_{C_{j}}\left(c_{j}(x)\right)$. Since the notion of probability density can be seen in the finite dimensional case as the limit of $P(\|X-x\| \leq h) / h$ when $h$ tends to $0,[17]$ suggests the use of $\prod_{j=1}^{q} f_{C_{j}}\left(c_{j}(x)\right)$ as an approximation for the density of $X$. In the sequel we give some additional justifications to this approximation.

Moreover, observe that we have, $\forall h>0, x \in L_{2}[0, T]$,

$P\left(\left\|X^{(q)}-x\right\| \leq h-\left\|X-X^{(q)}\right\|\right) \leq P(\|X-x\| \leq h) \leq P\left(\left\|X^{(q)}-x\right\| \leq h+\left\|X-X^{(q)}\right\|\right)$.

The relation (3) and (5) also suggest that the probability $P(\|X-x\| \leq h)$ could be approximated by $P\left(\left\|X^{(q)}-x\right\| \leq h\right)$.

Let $f_{X}^{(q)}$ denotes the joint probability density of $C^{(q)}=\left(C_{1}, \ldots, C_{q}\right)$. If $x=$ $\sum_{j \geq 1} c_{j}(x) \psi_{j}$ and $x^{(q)}=\sum_{j=1}^{q} c_{j}(x) \psi_{j}$ then

$$
P\left(\left\|X^{(q)}-x\right\| \leq h\right)=\int_{\mathcal{D}_{x}^{(q)}} f_{X}^{(q)}(y) d y
$$

where $\mathcal{D}_{x}^{(q)}=\left\{y \in \mathbb{R}^{q}:\left\|y-x^{(q)}\right\|_{\mathbb{R}^{q}} \leq \sqrt{h^{2}-\sum_{j \geq q+1} c_{j}^{2}(x)}\right\}$. The equation (5) and (6) suggest that the density $f_{X}^{(q)}$ can then be used as an approximation of the density of $X$. Moreover, when $X$ is a Gaussian process, the principal components $C_{j}$ are Gaussian and independent. The density $f_{X}^{(q)}$ is then:

$$
f_{X}^{(q)}(x)=\prod_{j=1}^{q} f_{C_{j}}\left(c_{j}(x)\right)
$$

with $f_{C_{j}}$ the Gaussian centred density of variance $\lambda_{j}$.

These results justify at least theoretically, the use of the principal component densities $f_{C_{j}}$ to approximate the notion of probability density of $X$. In 
particular, it gives a theoretical justification to the method $k C F C$ [11] which applies k-means on the principal components.

\section{Model-based clustering for functional data}

Several clustering algorithms for functional data used a truncation of the Karhunen-Loeve expansion [11, 22]. In these works, the truncation is used in order to define a distance between function, which relies on the difference between the first Karhunen-Loeve expansion coefficients. The approximation provided in (7) allows to define more general model-based clustering by considering that the observed curves re sampled from a mixture of such densities.

Let us consider that there exists a latent group variable $Z$, of $K$ modalities ( $K$ groups), such that $Z=Z_{1}, \ldots, Z_{K}$ with $Z_{g}=1$ if $X$ belongs to the cluster $g, 1 \leq g \leq K$, and 0 otherwise. Conditionally on $Z_{g}=1$, let assume that $X$

is a Gaussian random variable of density $f_{X_{\mid Z_{g}=1}}^{\left(q_{g}\right)}(x)$. Here, $q_{g}$ is the number of principal components used to approximate the density of $X$ conditionally on the group $g\left(Z_{g}=1\right)$. For each $i=1, \ldots, n$, let associate to $X_{i}$ the corresponding categorical variable $Z_{i}$ indicating the group $X_{i}$ belongs.

\subsection{The mixture model}

Let assume that each couple $\left(X_{i}, Z_{i}\right)$ is an independent realization of the random vector $(X, Z)$. Given a group $Z_{g}=1$, we consider the approximation (7) of the density of $X_{\mid Z_{g}=1}$ being:

$$
f_{X_{\mid Z_{g}=1}}^{\left(q_{g}\right)}\left(x ; \Sigma_{g}\right)=\prod_{j=1}^{q_{g}} f_{C_{j_{\mid Z_{g}=1}}}\left(c_{j, g}(x) ; \lambda_{j, g}\right)
$$

where $q_{g}$ is the number of the first principal components retained in the approximation (7) for the group $g, c_{j, g}(x)$ is the $j$ th principal component score of $X_{\mid Z_{g}=1}$ for $X=x, f_{C_{j, g}}$ its probability density and $\Sigma_{g}$ is the diagonal matrix $\operatorname{diag}\left(\lambda_{1, g}, \ldots, \lambda_{q_{g}, g}\right)$. Conditionally on the group, the probability density $f_{C_{j, g}}$ of the $j$ th principal component of $X$ is assumed to be the univariate Gaussian density with zero mean (the principal component are centred) and variance $\lambda_{j, g}$. This assumption is satisfied when $X_{\mid Z_{g}=1}$ is a Gaussian process.

The vector $Z=\left(Z_{1}, \ldots, Z_{K}\right)$ is assumed to have one order multinomial distribution $\mathcal{M}_{1}\left(\pi_{1}, \ldots, \pi_{K}\right)$, where $\pi_{1}, \ldots, \pi_{K}$ are the mixing probabilities 
$\left(\sum_{g=1}^{K} \pi_{g}=1\right)$. Under this model it follows that the unconditional approximated density of $X$ is given by

$$
f_{X}^{(q)}(x ; \theta)=\sum_{g=1}^{K} \pi_{g} \prod_{j=1}^{q_{g}} f_{C_{j, g}}\left(c_{j, g}(x) ; \lambda_{j, g}\right)
$$

where $\theta=\left(\pi_{g}, \lambda_{1, g}, \ldots, \lambda_{q_{g}, g}\right)_{1 \leq g \leq K}$ have to be estimated and $q=\left(q_{1}, \ldots, q_{K}\right)$. By extrapolation of the finite dimensional setting, we define a pseudo likelihood by:

$$
l^{(q)}(\theta ; \underline{X})=\prod_{i=1}^{n} \sum_{g=1}^{K} \pi_{g} \prod_{j=1}^{q_{g}} \frac{1}{\sqrt{2 \pi \lambda_{j, g}}} \exp \left(-\frac{1}{2} \frac{C_{i, j, g}^{2}}{\lambda_{j, g}}\right)
$$

where $C_{i, j, g}=C_{j, g}\left(X_{i}\right)$ is the $j$ th principal score of the curve $X_{i}$ belonging to the group $g$.

\subsection{Parameter estimation}

In the unsupervised context the estimation of the mixture model parameters is not as straightforward as in the supervised context since the groups labels $Z_{i}$ are unknown. A classical way to maximise a mixture model likelihood when data are missing (here the clusters indicators $Z_{i}$ ) is to use the iterative EM algorithm [23]. In this work we use an EM-like algorithm including in the M step the computation of the principal components scores of each group and the selection of the group specific dimension $q_{g}$. Our EM-like algorithm consists in maximizing the pseudo completed log-likelihood

$$
L_{c}^{(q)}(\theta ; \underline{X}, \underline{Z})=\sum_{i=1}^{n} \sum_{g=1}^{K} Z_{i, g}\left(\log \pi_{g}+\sum_{j=1}^{q_{g}} \log f_{C_{j, g}}\left(C_{i, j, g}\right)\right)
$$

which is easier to maximise than its incomplete version (9), and leads to the same estimate. Let $\theta^{(h)}$ be the current value of the estimated parameter at step $h, h \geq 1$.

$E$ step. As the group indicators $Z_{i, g}$ 's are unknown, the E step consists in computing the conditional expectation of the pseudo completed log-likelihood: 
$\mathcal{Q}\left(\theta ; \theta^{(h)}\right)=E_{\theta^{(h)}}\left[L_{c}^{(q)}(\theta ; \underline{X}, \underline{Z}) \mid \underline{X}=\underline{x}\right]=\sum_{i=1}^{n} \sum_{g=1}^{K} \tau_{i, g}\left(\log \pi_{g}+\sum_{j=1}^{q_{g}} \log f_{C_{j, g}}\left(c_{i, j, g}\right)\right)$

where $\tau_{i, g}$ is the probability for the curve $X_{i}$ to belong to the group $g$ conditionally to $C_{i, j, g}=c_{i, j, g}$ :

$$
\tau_{i, g}=E_{\theta^{(h)}}\left[Z_{i, g} \mid \underline{X}=\underline{x}\right] \simeq \frac{\pi_{g} \prod_{j=1}^{q_{g}} f_{C_{j, g}}\left(c_{i, j, g}\right)}{\sum_{l=1}^{K} \pi_{l} \prod_{j=1}^{q_{l}} f_{C_{j, l}}\left(c_{i, j, l}\right)} .
$$

The approximation (10) is due to the use of the approximation of the density of $X$ given by (7).

$M$ step. The M step is composed of three stages:

1. Principal score update.

The principal components $C_{j, g}$ of group $g$ are computed by weighting the curves according to the conditional probabilities $\tau_{i, g}(1 \leq i \leq n)$ computed in the E step. The estimation of the principal components is described in Section 3.3.

2. Group specific dimension $q_{g}$ selection.

The estimation of the group specific dimension $q_{g}$ is an open problem. In this work we propose to use, once the group specific FPCA have been computed, the scree-test of Cattell [24] in order to select each group specific dimension $q_{g}$. The advantage of using this test is that one hyperparameter (the threshold of the Cattell scree-test) allows to estimate $K$ approximation orders.

3. Parameters update.

The M step consists in computing the mixture model parameters $\theta^{(h+1)}$ which maximizes $\mathcal{Q}\left(\theta ; \theta^{(h)}\right)$. The variance $\lambda_{j, g}$ of the $j$ th principal component for cluster $g$ has already been computed in the principal score update step. For the mixing proportions, the usual estimator is obtained:

$$
\pi_{g}^{(h+1)}=\frac{1}{n} \sum_{i=1}^{n} \tau_{i, g}
$$

Let recall that the mean of the principal component $C_{j, g}$ is not consid- 
ered since it is 0 . The average shape of the curves of a cluster is taken into account in the computing of the principal components $C_{j, g}$ of the cluster.

Stopping criterion. When using an EM algorithm, usual stopping criterion is based on the growth of the likelihood. In our work, since the group specific approximation orders can change between two steps of the algorithm, the likelihood can artificially change (increase or decrease). In practice, we notice quite often that the estimation algorithm is hesitating between approximation orders, which prevents convergence of the pseudo likelihood. For this reason, the algorithm often stops on the maximum number of iterations allowed. In this case, the retained solution is the solution maximizing the pseudo-likelihood.

The proposed mixture model and the corresponding estimation algorithm will be called Funclust in what follows.

\subsection{Estimation and approximation for functional principal component anal- ysis (FPCA)}

Except some theoretical models (e.g. Brownian motion, Poisson process), the mean and the covariance function of the stochastic process $X$ are unknown. They are estimated from an i.i.d. sample of $X,\left\{X_{1}, \ldots, X_{n}\right\}$, $n>1$, by

$$
\hat{\mu}(t)=\frac{1}{n} \sum_{i=1}^{n} X_{i}(t), t \in[0, T]
$$

and

$$
\widehat{\operatorname{Cov}}(t, s)=\frac{1}{n-1} \sum_{i=1}^{n}\left(X_{i}(t)-\hat{\mu}(t)\right)\left(X_{i}(s)-\hat{\mu}(s)\right) .
$$

In the context of functional principal components, the asymptotic properties of these estimators are studied in [25] and [3]. Under the existence condition of fourth moment of $X$, in [26] are provided convergences rates for the estimators of the eigenvalues and of the eigenfunction of the integral operator with kernel $\widehat{\operatorname{Cov}}(t, s)$. See also [27] for more details.

\subsubsection{Smoothing and interpolating curves}

In practice, a new problem appears because of the continuous-time feature of the $X_{i}^{\prime}$ s. In practice, a curve $X_{i}=\left\{X_{i}(t), t \in[0, T]\right\}$ is usually observed 
only is a discrete set of time-points, $\left\{X\left(t_{i, s}\right), 0 \leq s \leq m_{i}, t_{i, s} \in[0, T]\right\}$, that is, we have only discrete observations of each sample path $X_{i}$ at a discrete set of knots $\left\{t_{i, s}: s=1, \ldots, m_{i}\right\}$. Because of this, the first step in functional data analysis is often the reconstruction of the functional form of data from discrete observations. In [28] it is shown that this is equivalent to the choice of a metric in the space of discrete observations. The most common solution to this problem is to consider that sample paths belong to a finite dimensional space of functions spanned by a basis of functions $\left\{\phi_{j}\right\}_{j=1, \ldots p}$ (see, for example, [5]).

$$
X_{i}(t)=\sum_{j=1}^{p} \gamma_{i, j} \phi(t), p \geq 1
$$

An alternative way of solving this problem is based on nonparametric smoothing of functions (see [20]).

Sample paths basis coefficients $\gamma_{i, j}$ 's are estimated from discrete-time observations by using an appropriate numerical method. If the functional predictor is observed with error,

$$
X_{i}^{o b s}\left(t_{i, s}\right)=X_{i}\left(t_{i, s}\right)+\varepsilon_{i s} \quad s=0, \ldots, m_{i}
$$

least square smoothing is used after choosing a suitable basis, for example, trigonometric functions, B-splines or wavelets (see [5] for a detailed study). In this case, the basis coefficients of each sample path $X_{i}$ are approximated by

$$
\widehat{\gamma}_{i}=\left(\Theta_{i}^{\prime} \Theta_{i}\right)^{-1} \Theta_{i}^{\prime} X_{i}^{o b s},
$$

with $\Theta_{i}=\left(\phi_{j}\left(t_{i s}\right)\right)_{1 \leq i \leq n, 1 \leq s \leq m_{i}}$ and $X_{i}^{o b s}=\left(X_{i}^{o b s}\left(t_{i, 0}\right), \ldots, X_{i}^{o b s}\left(t_{i, m_{i}}\right)\right)^{\prime}$.

The choice of the basis functions as well as the dimension of this basis are quite subjective. If the sample paths of $X$ are smooth and periodic then Fourier basis could be a good choice. However, the optimal properties of cubic B-spline functions make them the first choice for smoothing noisy data. See for example the monograph [29] and, in the context of functional data, see $[5]$.

If the sample curves are observed without error, an interpolation procedure can be used. For example, in [30] cuasi-cubic spline interpolation for reconstructing annual temperatures curves from monthly values is proposed. More about interpolation of functional data is provided in [21]. 


\subsubsection{FPCA computation}

Let $\Gamma$ be the $n \times p$ expansion coefficients $\gamma_{i j}$ matrix and $W$ be the matrix of the inner products between the basis functions $w_{j \ell}=\int_{0}^{T} \phi_{j}(t) \phi_{\ell}(t) d t$ $(1 \leq j, \ell \leq p)$. We explain here the computation of the principal component $C_{j, g}$ of group $g$ appearing in the M step previously describe. This computation is carried out by weighting the importance of each curve in the construction of the principal components with the conditional probabilities $T_{g}=\operatorname{diag}\left(\tau_{1, g}, \ldots, \tau_{n, g}\right)$. Consequently, the first step consists in centring the curve $X^{i}$ within the group $g$ by subtraction of the mean curve computed using the $\tau_{i, g}$ 's. The expansion coefficients of the centred curves are given by:

$$
\Gamma_{g}=\left(I_{n}-\mathbb{1}_{n}\left(\tau_{1, g}, \ldots, \tau_{n, g}\right)\right) \Gamma,
$$

where $I_{n}$ and $\mathbb{I}_{n}$ are respectively the identity $n \times n$-matrix and the unit $n$ vector. The $j$ th principal component scores $C_{j, g}$ is then the $j$ th eigenvector of the matrix $\Gamma_{g} W \Gamma_{g}^{\prime} T_{g}$ associated to the $j$ th eigenvalue $\lambda_{j, g}$ :

$$
\Gamma_{g} W \Gamma_{g}^{\prime} T_{g} C_{j, g}=\lambda_{j, g} C_{j, g} .
$$

Note that usual FPCA computation occurs if $T_{g}=\frac{1}{n} I_{n}$.

\section{Applications}

\subsection{Clustering evaluation}

Before validating the proposed clustering method on numerical applications, we have to choose an evaluation strategy, which remains an open questions in clustering. In lot of works, classification benchmark datasets are commonly used to validate and compared clustering models (see for instance $[3,31,32])$. As mentioned in several works [33, 34], this strategy can be sometimes dangerous and misleading. Indeed, this evaluation strategy relies on the assumption that class labels coincide with cluster structure, which can be true for some datasets and not for others. Another strategy can be the use of artificial datasets. But this strategy can also be criticized, since it evaluates the clustering only under particular assumption on the data generating process. [35] argues that the best way to evaluate clustering is probably to work on real world datasets, and to explain how the obtained clusters make sense. 
In this section, each of these three strategies will be used. First, a simulation study will be carried out to compare Funclust with two challengers for functional data clustering as well as usual clustering methods for finite dimensional data applied on FPCA scores. In a second part, the comparison is based on three classification datasets. Finally, a clustering of the surface of the soil of Mars will be estimated with Funclust, and a physical interpretation of the clusters will be used to validate the usefulness of the obtained clustering.

\subsection{Simulation study}

In this simulation, the number of clusters is assumed to be known: $K=2$. A sample of $n=100$ curves are simulated according to the following model inspired by $[36,37]$ :

$$
\begin{array}{lll}
\text { Class } 1: & X(t)=U_{1} h_{1}(t)+U_{2} h_{2}(t)+\epsilon(t), \quad t \in[1,21], \\
\text { Class } 2: & X(t)=U_{1} h_{1}(t)+\epsilon(t), \quad t \in[1,21],
\end{array}
$$

where $U_{1}$ and $U_{2}$ are independent Gaussian variables such that $\mathbb{E}\left[U_{1}\right]=$ $\mathbb{E}\left[U_{2}\right]=0, \operatorname{Var}\left(U_{1}\right)=\operatorname{Var}\left(U_{2}\right)=1 / 12$ and $\epsilon(t)$ is a white noise, independent of $U_{i}$ 's and such that $\operatorname{Var}\left(\epsilon_{t}\right)=1 / 12$. The function $h_{1}$ and $h_{2}$ are defined, for $t \in[1,21]$, by $h_{1}(t)=6-|t-7|$ and $h_{2}(t)=6-|t-15|$. The mixing proportions $\pi_{i}$ 's are choosen to be equal, and the curves are observed in 41 equidistant points $(t=1,1.5, \ldots, 21)$. Figure 1 plots the simulated curves. The functional form of the data is reconstructed using linear spline smoothing (with 30 equidistant knots).

Funclust is compared with two challengers for functional data clustering, FunHDDC [16] and fclust [13], and three clustering methods traditionally devoted to clustering finite-dimensional data applied on the FPCA scores: Gaussian mixture models on the FPCA scores (GMM, [4]) via the Rmixmod package for $\mathbf{R}$, k-means [2] and hierarchical clustering (packages kmeans and hclust). The selection of the number of FPCA components is carried out by the Cattell scree test. For FunHDDC and GMM, which proposes several

models, the best model according to BIC has been retained. Figure 2 shows the correct classification rates over 100 simulations, which exhibited better results for Funclust on this simulation set-up. 

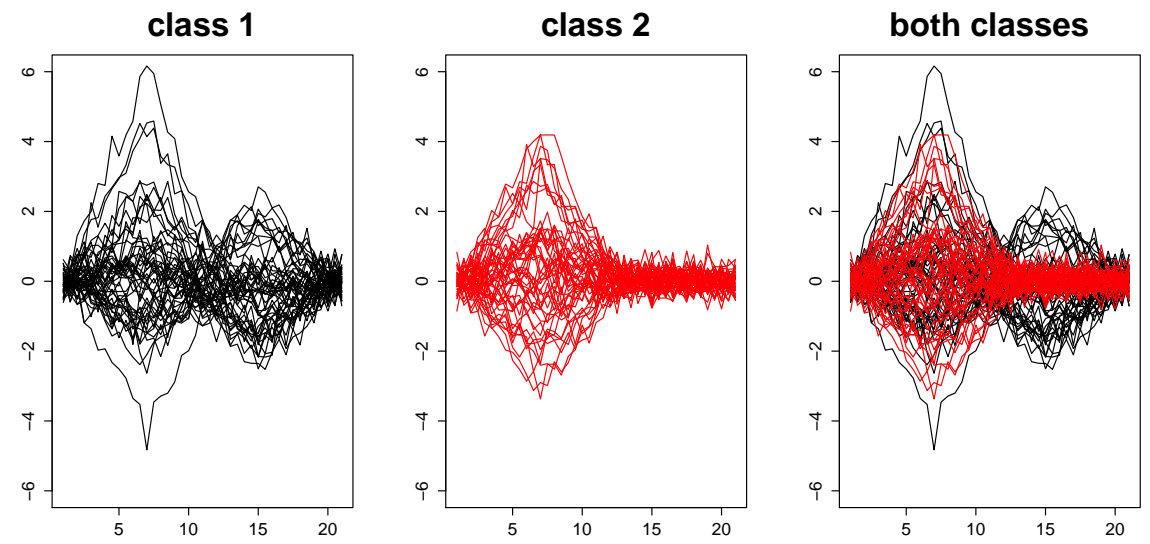

Figure 1: Class 1 (left), Class 2 (center) and both classes (right).

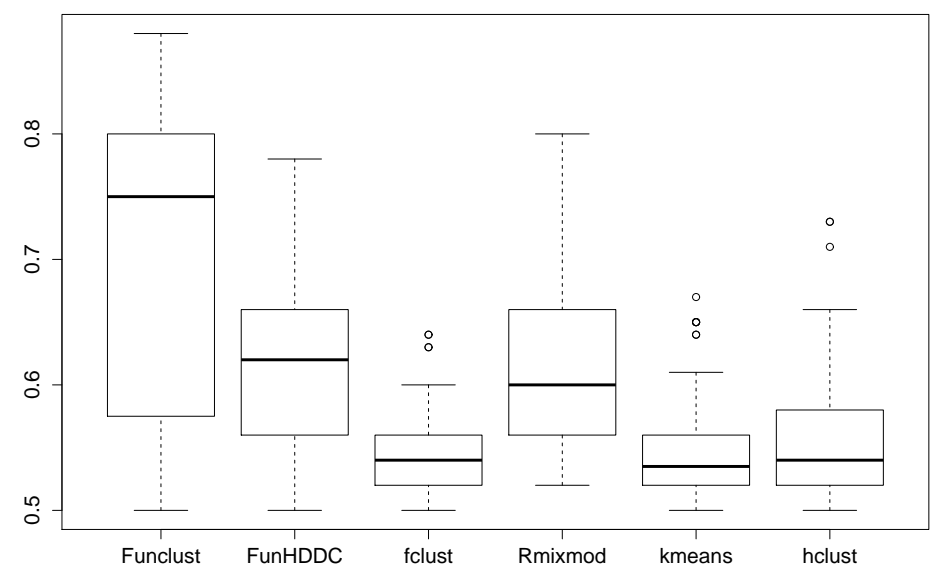

Figure 2: Correct classification rates over 100 simulations.

\subsection{Benchmark study}

Funclust is now compared with other clustering methods on the basis of the capacity to find the class labels of three classification datasets.

\subsubsection{The data}

Three real datasets are considered: the Kneading, Growth, and ECG datasets. These three datasets are plotted in Figure 3. The Kneading dataset 

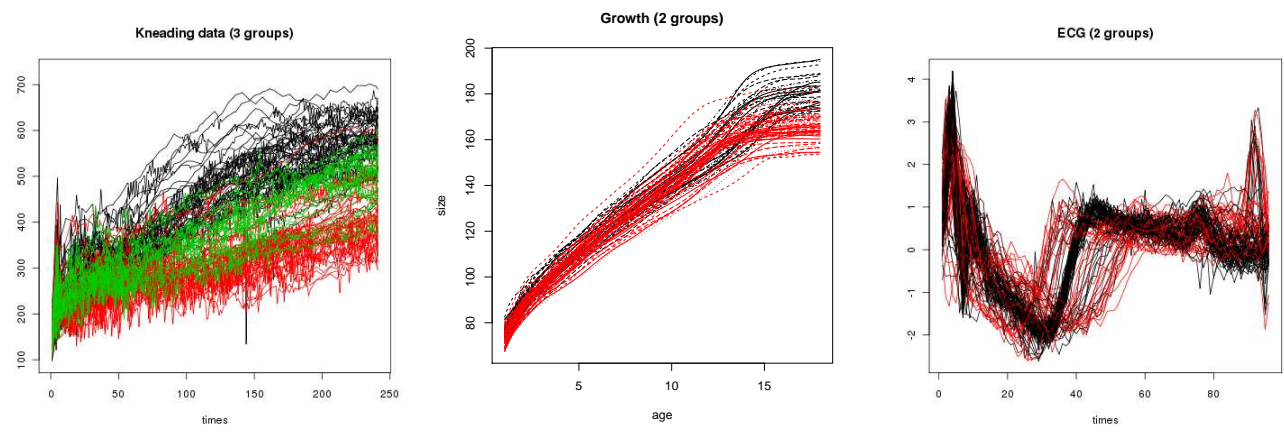

Figure 3: Kneading, Growth and ECG datasets.

comes from Danone Vitapole Paris Research Center and concerns the quality of cookies and the relationship with the flour kneading process. The kneading dataset is described in detail in [38]. There are 115 different flours for which the dough resistance is measured during the kneading process for 480 seconds. One obtains 115 kneading curves observed at 241 equispaced instants of time in the interval $[0,480]$. The 115 flours produce cookies of different quality: 50 of them have produced cookies of good quality, 25 produced medium quality and 40 low quality. This data, have been already studied in a supervised classification context $[38,39]$. They are known to be hard to discriminate, even for supervised classifiers, partly because of the medium quality class. Taking into account that the resistance of dough is a smooth curve measured with error, and following previous works on this data [38, 39], least squares approximation on a basis of cubic B-spline functions (with 18 knots) is used to reconstruct the true functional form of each sample curve.

The Growth dataset comes from the Berkeley growth study [40] and is available in the $f d a$ package of $\mathbf{R}$. In this dataset, the heights of 54 girls and 39 boys were measured at 31 stages, from 1 to 18 years. The goal is to cluster the growth curves and to determine whether the resulting clusters reflect gender differences. The ECG dataset is taken from the UCR Time Series Classification and Clustering website ${ }^{1}$. This dataset consists of 200 electrocardiogram from 2 groups of patients sampled at 96 time instants, and has already been studied in [41]. For these two datasets, the same basis functions as for the Kneading dataset has been arbitrarily chosen (20 cubic B-splines).

\footnotetext{
${ }^{1}$ http://www.cs.ucr.edu/ eamonn/time_series_data/
} 


\subsubsection{Experimental setup}

In this benchmark study, Funclust is compared with FunHDDC and fclust, as in the simulation study. The Growth dataset allows an additional comparison with $k$-centres (kCFC, [11]), since they present in [11] the correct classification rate they obtained on the Growth dataset (their code are not available to the best of our knowledge). Concerning the finite-dimensional methods to which Funclust is compared, we added to GMM, k-means and hierarchical clustering, two methods dedicated to the clustering of high-dimensional data: $H D D C$ [7] and MixtPPCA [6] (HDclassif package). These methods for finite-dimensional data have been applied on the FPCA scores with choice of the number of components with the Cattell scree test, but also directly on the discrete observations of the curves and on the coefficients in the cubic $B$-spline basis approximation.

Details for Funclust. The maximum number of iterations is fixed to 200. Note that for these three applications, the maximum number of iterations has always been reached. Nevertheless, since the iterations corresponding to the retained solutions (according to the best pseudo-likelihood) were always relatively far from the last one, we assume this maximum number of iterations as sufficient. The threshold of the Cattell scree test allowing to select the approximation order $q_{k}$ is fixed to 0.05 . In order to avoid convergence to a local maximum of the pseudo likelihood, our EM-like algorithm has been initialized with the best solutions of 20 small EM-like algorithms with 20 iterations each [42]. With this experimental set-up, Funclust estimation is obtained in about 30 seconds for each dataset, on a laptop $(2.80 \mathrm{GHz} \mathrm{CPU})$ and with a code in $\mathbf{R}$ software.

\subsubsection{Results}

The estimated approximation order $q_{g}$ for Funclust are the following: Kneading $\left(q_{1}=2, q_{2}=1, q_{3}=3\right)$, Growth $\left(q_{1}=2, q_{2}=3\right)$, ECG $\left(q_{1}=9, q_{2}=4\right)$. The correct classification rates $(\mathrm{CCR})$ according to the known partitions are given in Table 1. Funclust performs better to estimate the class label than all the other methods on two datasets among three (Kneading and ECG). On the last dataset, the results are relatively poor (69.89\% accurate whereas some method are about $97 \%$ accurate), but the performance can be greatly increased (95.70\%) if the dimensions $q_{g}$ are fixed to 2 (as the number of FPCA scores used by the non functional methods). This dataset illustrates that the choice of the approximation order is a very 


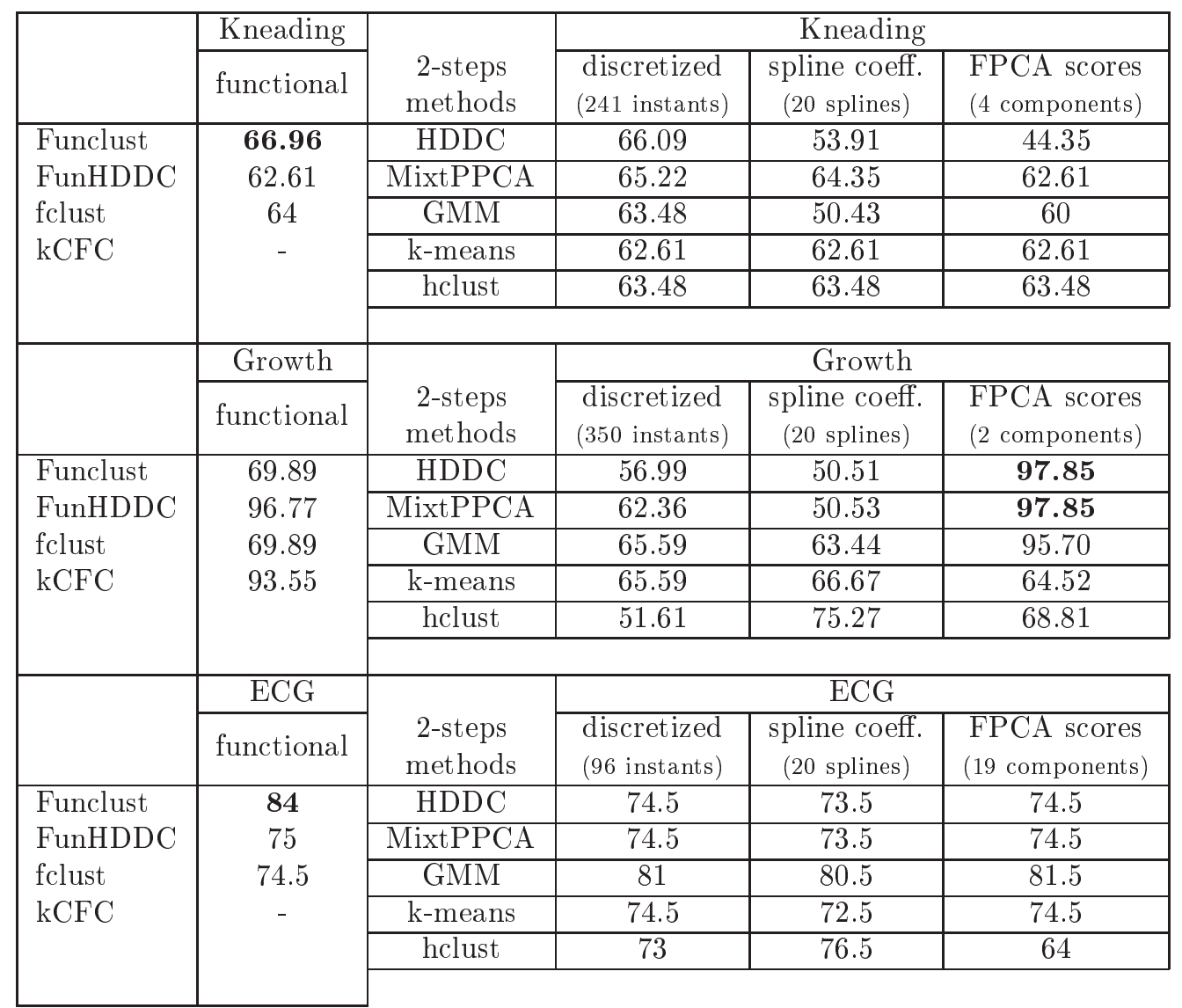

Table 1: Correct classification rates (CCR) in percentage for Funclust, FunHDDC (best model according $\mathrm{BIC}$ ), fclust, $\mathrm{kCFC}$ and usual non-functional methods on the Kneading, Growth and ECG datasets. 
important question, and that further works have to be carried out in this direction. A last remark concerns the use of non functional methods. These methods can sometimes perform very well to estimate the class label, but the main problem is that, in the present unsupervised context, we have no way to choose between working with the discrete data, with the spline coefficients or with the FPCA scores. For instance, HDDC and MixtPPCA are very well performing on the Growth dataset using the FPCA scores, but they are very poor using the discrete data or the spline coefficients.

\subsection{Application to Mars surface characterization}

This data, provided by the Laboratory of Planetology of Grenoble [43, 44], were acquired by the OMEGA imaging satellite. The soil of Mars has been observed with a resolution between 300 and 3000 meters depending on the altitude of the satellite. It was acquired for each pixel a spectra whose wavelengths range from 0.36 to 5.2 microns and stored this information in a vector of 256 dimensions. The purpose of this preliminary study is to characterize the composition of the surface of Mars by determining zones composed of similar material. The number of groups has been fixed to 8, since the experts expect 8 main classes of mineralogical. The analysis of some spectra in each cluster will allow the expert to indicate to which mineralogical corresponds each cluster. A photography of size $300 \times 128$ pixels of the surface of Mars (left image of Figure 4) is considered, each of 38,400 pixels being described by a spectrum (right image of Figure 4).
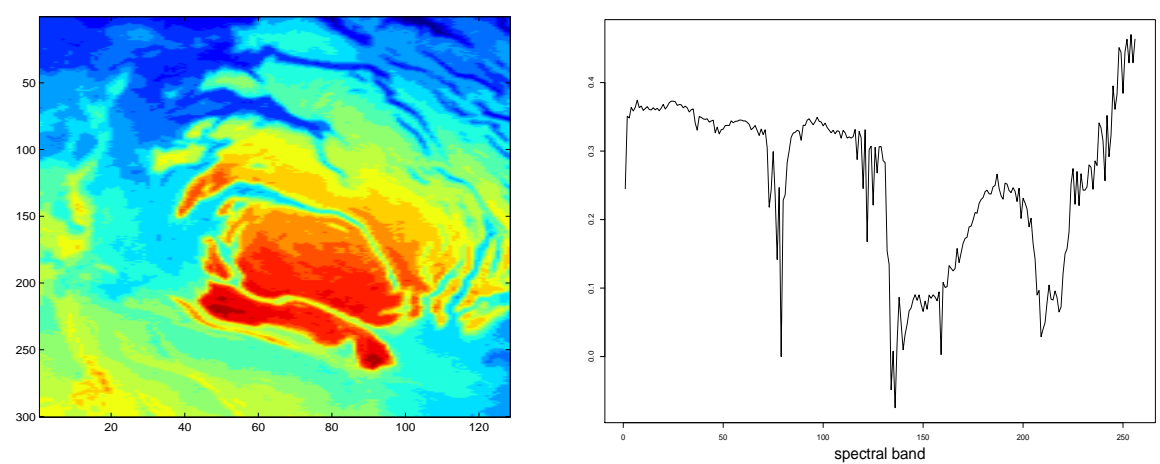

Figure 4: Mars data: image of the studied zone. 


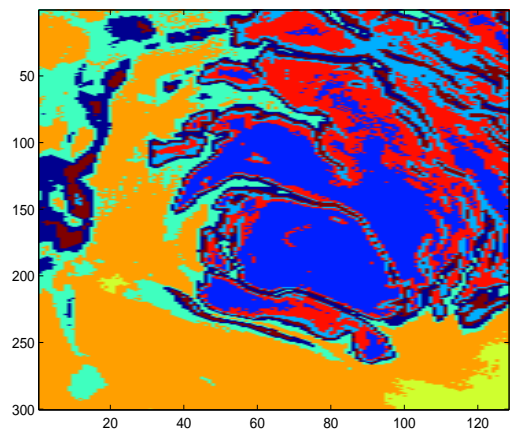

Figure 5: Funclust clustering in 8 groups.

The clusters resulting from Funclust is represented in Figure 5. This clustering seems to be in accordance with the photography of Figure 4) (we recall that no spatial information has been used for this clustering). Moreover, the experts of the Laboratory of Planetology of Grenoble particularly appreciated that our method is able to detect specific cluster, in the form of edging, at the border of the main areas: for instance the magenta and cyan clusters separate the main blue and orange area. Analysing some spectra in each cluster has allowed to deduce that these clusters reflect the presence of particular materials (mixture of carbonate and ice) at the border of main materials (ice and dust).

\section{Conclusion}

In this paper we propose a new clustering procedure for functional data based on an approximation of the notion of density of a random function. The main tool is the use of the probability densities of the principal components scores. Assuming that the functional data are sampled from a Gaussian process, the resulting mixture model is an extrapolation of the finite dimensional Gaussian mixture model to the infinite dimensional setting. We defined an EM-like algorithm for the parameter estimation and performed several numerical applications, in order to show the performance of this approach with respect to usual clustering procedures.

Future work is devoted to investigate the choice of the approximation orders. We observed in our application study that a bad choice of these dimension 
can drastically deteriorate the clustering results. However, allowing the approximation order to change in the estimation algorithm leads to loose the properties of the EM algorithm. In particular, the pseudo likelihood is not necessarily increasing, and we have to stop the algorithm after a given number of iterations and to choose the best reached solutions.

\section{References}

[1] J. H. Ward, Jr., Hierarchical grouping to optimize an objective function, J. Amer. Statist. Assoc. 58 (1963) 236-244.

[2] J. Hartigan, M. Wong, Algorithm as 1326 : A k-means clustering algorithm, Applied Statistics 28 (1978) 100-108.

[3] J. Banfield, A. Raftery, Model-based gaussian and non-gaussian clustering, Biometrics 49 (1993) 803-821.

[4] G. Celeux, G. Govaert, Gaussian parsimonious clustering models, The Journal of the Pattern Recognition Society 28 (1995) 781-793.

[5] J. O. Ramsay, B. W. Silverman, Functional data analysis, Springer Series in Statistics, Springer, New York, second edition, 2005.

[6] M. E. Tipping, C. Bishop, Mixtures of principal component analyzers, Neural Computation 11 (1999) 443-482.

[7] C. Bouveyron, S. Girard, C. Schmid, High Dimensional Data Clustering, Computational Statistics and Data Analysis 52 (2007) 502-519.

[8] J. Jacques, C. Bouveyron, S. Girard, O. Devos, L. Duponchel, , C. Ruckebusch, Gaussian mixture models for the classification of highdimensional vibrational spectroscopy data, Journal of Chemometrics 24 (2010) 719-727.

[9] C. Abraham, P. A. Cornillon, E. Matzner-Løber, N. Molinari, Unsupervised curve clustering using B-splines, Scand. J. Statist. 30 (2003) 581-595.

[10] T. Tarpey, K. Kinateder, Clustering functional data, J. Classification 20 (2003) 93-114. 
[11] J.-M. Chiou, P.-L. Li, Functional clustering and identifying substructures of longitudinal data, J. R. Stat. Soc. Ser. B Stat. Methodol. 69 (2007) 679-699.

[12] A. Antoniadis, X. Brossat, J. Cugliari, J.-M. Poggi, Clustering functional data using wavelets, Rapport de recherche RR-7515, INRIA, 2011.

[13] G. James, C. Sugar, Clustering for sparsely sampled functional data, J. Amer. Statist. Assoc. 98 (2003) 397-408.

[14] S. Ray, B. Mallick, Functional clustering by Bayesian wavelet methods, J. R. Stat. Soc. Ser. B Stat. Methodol. 68 (2006) 305-332.

[15] S. Frühwirth-Schnatter, S. Kaufmann, Model-based clustering of multiple time series, Journal of Business and Economic Statistics 26 (2008) 78-89.

[16] C. Bouveyron, J. Jacques, Model-based clustering of time series in group-specific functional subspaces, Advances in Data Analysis and Classification 5 (2011) 281-300.

[17] A. Delaigle, P. Hall, Defining probability density for a distribution of random functions, The Annals of Statistics 38 (2010) 1171-1193.

[18] G. Celeux, G. Govaert, A classification EM algorithm for clustering and two stochastic versions, Comput. Statist. Data Anal. 14 (1992) 315-332.

[19] A. P. Dempster, N. M. Laird, D. B. Rubin, Maximum likelihood from incomplete data via the EM algorithm, J. Roy. Statist. Soc. Ser. B 39 (1977) 1-38.

[20] F. Ferraty, P. Vieu, Nonparametric functional data analysis, Springer Series in Statistics, Springer, New York, 2006.

[21] G. Saporta, Méthodes exploratoires d'analyse de données temporelles, Cahiers du Buro 37-38 (1981).

[22] J. Peng, H.-G. Müller, Distance-based clustering of sparsely observed stochastic processes, with applications to online auctions, Ann. Appl. Stat. 2 (2008) 1056-1077. 
[23] G. McLachlan, D. Peel, Finite Mixture Models, Wiley Interscience, New York, 2000.

[24] R. Cattell, The scree test for the number of factors, Multivariate Behav. Res. 1 (1966) 245-276.

[25] J. Dauxois, A. Pousse, Y. Romain, Asymptotic theory for the principal component analysis of a vector random function: some applications to statistical inference, Journal of multivariate analysis 12 (1982) 136-154.

[26] J. Deville, Méthodes statistiques et numériques de l'analyse harmonique, Annales de l'INSEE (1974) 3-101.

[27] J. Rice, B. Silverman, Estimating the mean and covariancee structure nonparametrically when the data are curves, Journal of the Royal Statistical Society (B) 53 (1991) 233-243.

[28] P. Besse, Approximation spline et optimalité en Analyse en Composantes Principales, Ph.D. thesis, Université Toulouse III, 1989.

[29] C. De Boor, A practical guide to Splines, Springer, 2001.

[30] M. Escabias, A. Aguilera, M. Valderrama, Principal component estimation of functional logistic regression: discussion of two different approaches, Journal of Nonparametric Statistics 16 (2004) 365-384.

[31] J. Baek, G. McLachlan, L. Flack, Mixtures of Factor Analyzers with Common Factor Loadings: Applications to the Clustering and Visualization of High-Dimensional Data, IEEE Transactions on Pattern Analysis and Machine Intelligence 32 (2010).

[32] T. Hastie, R. Tibshirani, J. Friedman, The elements of statistical learning, Springer Series in Statistics, Springer-Verlag, New York, second edition, 2009.

[33] I. Guyon, U. Von Luxburg, R. Williamson, Clustering: Science or art, in: NIPS 2009 Workshop on Clustering Theory.

[34] I. Färber, S. Günnemann, H. Kriegel, P. Kröger, E. Müller, E. Schubert, T. Seidl, A. Zimek, On using class-labels in evaluation of clusterings, in: MultiClust: 1st International Workshop on Discovering, Summarizing 
and Using Multiple Clusterings Held in Conjunction with KDD 2010, Washington, DC.

[35] J. Kogan, Introduction to Clustering Large and High-Dimensional Data, Cambridge University Press, 2007.

[36] F. Ferraty, P. Vieu, Curves discrimination: a nonparametric approach, Computational Statistics and Data Analysis 44 (2003) 161-173.

[37] C. Preda, Regression models for functional data by reproducing kernel hilbert spaces methods, Journal of Statistical Planning and Inference 137 (2007) 829-840.

[38] C. Leveder, C. Abraham, P. A. Cornillon, E. Matzner-Løber, N. Molinari, Discrimination de courbes de pétrissage, in: Chimiométrie 2004, Paris, pp. 37-43.

[39] C. Preda, G. Saporta, C. Lévéder, PLS classification of functional data, Comput. Statist. 22 (2007) 223-235.

[40] R. Tuddenham, M. Snyder, Physical growth of california boys and girls from birth to eighteen years, Univ. Calif. Publ. Chld Devlpmnt 1 (1954) 188-364.

[41] R. Olszewski, Generalized Feature Extraction for Structural Pattern Recognition in Time-Series Data, Ph.D. thesis, Carnegie Mellon University, Pittsburgh, PA, 2001.

[42] C. Biernacki, Initializing EM using the properties of its trajectories in Gaussian mixtures, Statistics and Computing 14 (2004) 267-279.

[43] J.-P. Bibring, 42 co-authors, OMEGA: Observatoire pour la Minéralogie, l'Eau, les Glaces et l'Activité, ESA SP-1240: Mars Express: the Scientific Payload, p. 3749.

[44] C. Bernard-Michel, S. Douté, M. Fauvel, L. Gardes, S. Girard, Retrieval of Mars surface physical properties frim OMEGA hyperspectral images using regularized sliced inverse regression, Journal of Geophysical Research 114 (2009) E06005. 